\title{
JOURNAL OF RHEOLOGY
}

Effect of upstream boundary conditions on stability of fiber spinning in the highly elastic limit

Michael Renardy

Citation: Journal of Rheology (1978-present) 46, 1023 (2002); doi: 10.1122/1.1487369

View online: http://dx.doi.org/10.1122/1.1487369

View Table of Contents: http://scitation.aip.org/content/sor/journal/jor2/46/4?ver=pdfcov

Published by the The Society of Rheology

\section{Re-register for Table of Content Alerts}


Copyright by the American Institute of Physics (AIP). Renardy, M. "effect of upstream boundary conditions on stability of fiber spinning in the highly elastic limit," J. Rheol. 46, 1023 (2002); http://dx.doi.org/10.1122/1.1487369

\title{
Effect of upstream boundary conditions on stability of fiber spinning in the highly elastic limit
}

\author{
Michael Renardy ${ }^{\text {a) }}$ \\ Department of Mathematics, Virginia Polytechnic Institute and State University, \\ Blacksburg, Virginia 24061-0123
}

(Received 25 January 2002; final revision received 26 April 2002)

\section{Synopsis}

We consider fiber spinning for the upper-convected Maxwell fluid in the limit of a high Deborah number. We compare several choices of boundary conditions that may be imposed. In addition to the takeup speed and the upstream flow rate, we consider four different boundary conditions: the upstream velocity, upstream elastic stress, the force in the fiber, and the ratio of stress to the square of the velocity (the latter can be motivated by a limit of vanishing retardation time). We find that the effect of the boundary condition on stability is crucial; in one case we even find an instability even though the draw ratio is 1 . (C) 2002 The Society of Rheology.

[DOI: $10.1122 / 1.1487369]$

\section{INTRODUCTION AND GOVERNING EQUATIONS}

We consider the fiber spinning problem for the upper-convected Maxwell fluid in the case of zero inertia and surface tension. We shall denote by $v$ the velocity, $r$ the radius of the fiber, and $T$ and $Y$ the axial and radial elastic stress. All equations are written in dimensionless form. The equations governing the problem are the conservation of mass

$$
\left(r^{2}\right)_{t}+\left(v r^{2}\right)_{x}=0 \text {, }
$$

conservation of momentum

$$
\left[r^{2}(T-Y)\right]_{x}=0,
$$

and the constitutive law for the upper-convected Maxwell (UCM) fluid

$$
\begin{aligned}
& T_{t}+v T_{x}-2 T v_{x}=\frac{1}{\operatorname{De}}\left(2 v_{x}-T\right), \\
& Y_{t}+v Y_{x}+Y v_{x}=\frac{1}{\mathrm{De}}\left(-v_{x}-Y\right) .
\end{aligned}
$$

Here De is the Deborah number. The length of the spin line is nondimensionalized to 1.

For boundary conditions, we prescribe the takeup speed,

$$
v(1, t)=1,
$$

the upstream flow rate,

\footnotetext{
a)Author to whom correspondence should be addressed; electronic mail: renardym@math.vt.edu
} 


$$
r^{2}(0, t) v(0, t)=Q_{0},
$$

the upstream radial elastic stress

$$
Y(0, t)=0
$$

and one of the following:

(1) The upstream velocity $v(0, t)=v_{0}$.

(2) The upstream axial stress $T(0, t)=T_{0}$.

(3) The force in the fiber: $T(0, t) r(0, t)^{2}=F_{0}$.

(4) The ratio of the stress to the square of the velocity; $T(0, t) / v(0, t)^{2}=P_{0}$.

The question of boundary conditions to be imposed on the one-dimensional fiber spinning model is by no means clear from a physical point of view, since the Maxwell demons who control the boundary conditions needed for mathematical analysis are conspicuously absent in real devices. With the exception of downstream condition (4), all the boundary conditions can be questioned. Even the location of the upstream boundary is not obvious. When the fluid leaves the spinneret, a transition from shear flow to elongational flow must take place, and one may think of the upstream boundary as the point where this transition is completed. Clearly, this point is ill defined and uncontrolled. In the high Deborah number limit, in particular, we are assuming that the fluid traverses the spin line in a time small compared to the relaxation time, while presumably the time required to complete the transition from shear to extensional flow must be of the order of the relaxation time.

We choose condition (5) because, at least in the steady case, the flow rate must be constant along the fiber and thus equal to the flow rate in the spinneret upstream (note, however, that even that may not be so dynamically). We choose condition (6) because, at high Deborah number, we expect the radial stress to be small relative to the axial stress, and hence, not significant. Also, condition (6) matches the flow in the spinneret upstream, since the second normal stress difference for the UCM fluid is zero [see, e.g., the discussion on p. 187 of Petrie (1979)]. The objective of this note is to explore the effect of the choice of the remaining boundary condition on stability. We shall find that all four cases are fundamentally different. Even the three cases that are stable are not similar: for prescribed velocity the eigenvalue governing stability tends to zero at infinite De, for prescribed ratio of stress to square of velocity it has a finite limit, and for prescribed force there are no eigenvalues at infinite De. For prescribed stress, finally, we find an unstable eigenvalue. Presumably, this sensitivity to the choice of boundary conditions would persist to lower values of the Deborah number as well. The advantage of analyzing the high Deborah limit is its mathematical simplicity, which allows the eigenvalues to be found explicitly. I note that sensitivity of stability to the choice of boundary conditions has been observed for multimode models (K. Christodoulou, private communication), but I am not aware of any published work on this.

An alternative way of interpreting the upstream boundary condition is to apply it at the exit of the spinneret (where the flow is not one-dimensional) and interpret the values of $v, T$, etc., as cross-sectional averages. Although this cannot really be justified, one might hope for some qualitative validity. If one chooses this interpretation, one should impose values of $v(0, t), r(0, t), T(0, t)$, and $Y(0, t)$ to match the upstream flow. The upperconvected Maxwell model, however, does not allow that many boundary conditions. We can resolve this dilemma by introducing a small retardation term. The effect of such a term is to change (2) to 


$$
\left[r^{2}\left(T-Y+3 \epsilon v^{\prime}\right)\right]_{x}=0
$$

while Eq. (3) remains the same. In particular, the first equation of Eq. (3) can be recast in the form

$$
v^{-3}\left(T_{t}+\frac{T}{\mathrm{De}}\right)+\left(\frac{T+1 / \mathrm{De}}{v^{2}}\right)_{x}=0 .
$$

In the limit $\epsilon \rightarrow 0$, a boundary layer occurs at the upstream boundary. When Eq. (8) is integrated across the boundary layer, we find that the jump in $(T+1 / \mathrm{De}) / v^{2}$ is of the order of the boundary layer thickness. As a consequence, $(T+1 / \mathrm{De}) / v^{2}$ must be constant across the boundary layer in the limit $\epsilon \rightarrow 0$. This motivates the fourth alternative of the boundary condition introduced above; we can think of this condition as a limit where we first introduce a small retardation time and prescribe both $T(0)$ and $v(0)$ and then let the retardation time tend to zero.

We are concerned with the limit of infinite Deborah number, i.e., we shall neglect the terms of order 1/De in Eq. (3). In this case, we obtain the constant steady-state solution

$$
v=1, \quad T=T_{0}, \quad r=r_{0},
$$

which needs to be consistent with the boundary conditions. For the case of prescribed upstream velocity, this imposes the restriction $v_{0}=1$. We note that the steady solution for the infinite Deborah number limit is actually a uniform flow in which the fiber is not stretched at all. This is consistent with the prior literature [Denn et al. (1975), Forest and Wang (1994), Petrie (1987), Tanner (2000)], where it was noted that the maximum attainable draw ratio is of the order $1+1 / \mathrm{De}$, i.e., in the limit $\mathrm{De} \rightarrow \infty$, the draw ratio must be 1 .

The question of stability in the high Deborah number limit does not seem to have been addressed in prior literature. Tanner's book [Tanner (2000)] discusses the steady state in the limit De $\rightarrow \infty$, but not stability. Stability studies in the literature [Forest and Wang (1994), Pearson (1985), Tanner (2000)] do not seem to address the limit De $\rightarrow \infty$. The boundary condition used in these prior works is that of prescribed velocity.

\section{LINEAR STABILITY}

We linearize at the steady solution and assume a time dependence proportional to $\exp (\sigma t)$. This yields the following equations for the infinite Deborah number limit:

$$
\begin{gathered}
2 \sigma r+2 r^{\prime}+r_{0} v^{\prime}=0, \\
2 T_{0} r^{\prime}+r_{0} T^{\prime}=0, \\
\sigma T+T^{\prime}-2 T_{0} v^{\prime}=0 .
\end{gathered}
$$


This system has the solution

$$
\begin{gathered}
r=c_{1}+c_{3} e^{-\sigma x}, \\
v=-\frac{2 c_{1} \sigma x}{r_{0}}+c_{2}, \\
T=\frac{-2 c_{3} T_{0}}{r_{0}} e^{-\sigma x}-\frac{4 c_{1} T_{0}}{r_{0}} .
\end{gathered}
$$

Two of the boundary conditions are

$$
v(1)=2 r(0)+r_{0} v(0)=0 .
$$

This leads to

$$
c_{2}=\frac{2 c_{1} \sigma}{r_{0}}, \quad c_{3}=-c_{1}(1+\sigma) .
$$

The third boundary condition yields the following:

(1) For prescribed stress $T(0)=0$, leading to $\sigma=1$.

(2) For prescribed force $T(0) r_{0}+2 T_{0} r(0)=0$, leading to no eigenvalues.

(3) For prescribed ratio of stress to square of the velocity $T(0)-2 T_{0} v(0)=0$, leading to $\sigma=-1$.

(4) For prescribed velocity $v(0)=0$, leading to $\sigma=0$.

Hence, we find instability for prescribed stress, stability for prescribed force, or prescribed ratio of stress to square of velocity, and neutral stability for prescribed velocity. For the latter case we, therefore, need to consider higher-order perturbations to decide stability. We investigate this situation in the next section.

\section{CASE OF PRESCRIBED VELOCITY}

It turns out that we need to consider terms up to order $1 / \mathrm{De}^{2}$ to decide stability. We begin with the steady state. The equations are

$$
\begin{gathered}
\left(r^{2} v\right)^{\prime}=0, \\
\left(r^{2}(T-Y)\right)^{\prime}=0, \\
v T^{\prime}-2 T v^{\prime}=\frac{1}{\mathrm{De}}\left(2 v^{\prime}-T\right), \\
v Y^{\prime}+Y v^{\prime}=\frac{1}{\mathrm{De}}\left(-v^{\prime}-Y\right),
\end{gathered}
$$

with boundary conditions

$$
v(1)=1, \quad Y(0)=0, \quad v(0) r(0)^{2}=r_{0}^{2}, \quad v(0)=1-\frac{\alpha}{\mathrm{De}}+\frac{\beta}{\mathrm{De}^{2}},
$$

where $\alpha$ and $\beta$ are determined as part of the solution. We expand each variable in a series in power of 1/De: 


$$
r=r_{0}+\frac{1}{\mathrm{De}} r_{1}(x)+\frac{1}{\mathrm{De}^{2}} r_{2}(x)+\cdots
$$

etc. The result of the calculation is

$$
\begin{gathered}
r(x)=\tilde{r}(x)=r_{0}\left(1+\frac{(1-x)}{2 \mathrm{De}}+\frac{1}{8 T_{0} \mathrm{De}^{2}}\left[8(x-1)+3 T_{0}(x-1)^{2}\right]\right), \\
v(x)=\tilde{v}(x)=1+\frac{1}{\mathrm{De}}(-1+x)+\frac{2}{T_{0} \mathrm{De}^{2}}(1-x), \\
T(x)=\tilde{T}(x)=T_{0}+\frac{T_{0} x}{\mathrm{De}}+\frac{\left(T_{0}-4\right) x}{\mathrm{De}^{2}}, \\
Y(x)=\tilde{Y}(x)=-\frac{x}{\mathrm{De}^{2}} .
\end{gathered}
$$

We note that at $\mathrm{De}=\infty$, we must have $v(0)=1$ in order to have a steady solution, but this steady solution is then undetermined since $T_{0}$ is arbitrary. In our perturbation series, we correspondingly hold $T(0)$ fixed (changing this value would merely be equivalent to changing $T_{0}$ ). The steady solution found here (up to terms of order $1 / \mathrm{De}$ ) agrees with Eq. (29) in Denn et al. (1975).

We now linearize at this steady solution and assume a perturbation proportional to $\exp (\sigma t)$. The resulting eigenvalue problem is as follows:

$$
\begin{gathered}
2 \sigma r+\tilde{r} v^{\prime}+r \tilde{v}^{\prime}+2 \tilde{v} r^{\prime}+2 v \tilde{r}^{\prime}=0, \\
2(\tilde{T}-\tilde{Y}) r^{\prime}+2(T-Y) \tilde{r}^{\prime}+\tilde{r}\left(T^{\prime}-Y^{\prime}\right)+r\left(\tilde{T}^{\prime}-\tilde{Y}^{\prime}\right)=0, \\
\sigma T+\tilde{v} T^{\prime}+v \tilde{T}^{\prime}-2 \tilde{T} v^{\prime}-2 T \tilde{v}^{\prime}+\frac{1}{\mathrm{De}}\left(T-2 v^{\prime}\right)=0, \\
\sigma Y+\tilde{v} Y^{\prime}+v \tilde{Y^{\prime}}+\tilde{Y} v^{\prime}+Y \tilde{v}^{\prime}+\frac{1}{\mathrm{De}}\left(Y+v^{\prime}\right)=0 .
\end{gathered}
$$

Boundary conditions are

$$
v(1)=v(0)=r(0)=Y(0)=0 \text {. }
$$

We expand the eigenvalue and eigenfunction in powers of 1/De. We omit the details of the calculation. The outcome is that the leading contribution is

$$
\sigma=-\frac{2}{T_{0} \mathrm{De}^{2}}
$$

leading to stability.

\section{ACKNOWLEDGMENT}

This research was supported by the National Science Foundation under Grant No. DMS-0103813. 


\section{References}

Denn, M. M., C. J. S. Petrie, and P. Avenas, "Mechanics of steady spinning of a viscoelastic liquid," AIChE J. 21, 791-799 (1975).

Forest, M. G. and Q. Wang, "Dynamics of slender viscoelastic free jets,” SIAM (Soc. Ind. Appl. Math.) J. Appl. Math. 54, 996-1032 (1994).

Pearson, J. R. A., Mechanics of Polymer Processing (Elsevier, Amsterdam, 1985).

Petrie, C. J. S., Elongational Flows (Pitman, London, 1979).

Petrie, C. J. S., "Boundary value problems in extensional flow," Fluid Dyn. Trans. 13, 171-204 (1987).

Tanner, R. I., Engineering Rheology, 2nd ed. (Oxford University Press, Oxford, U.K., 2000). 